$11-19-2014$

\title{
Leukocyte esterase strip test: matched for musculoskeletal infection society criteria.
}

\author{
Eric H Tischler \\ The Rothman Institute of Orthopedics at Thomas Jefferson University Hospital \\ Priscilla K Cavanaugh \\ Rothman Institute, Thomas Jefferson University \\ Javad Parvizi MD \\ Rothman Institute, Thomas Jefferson University
}

Follow this and additional works at: https://jdc.jefferson.edu/rothman_institute

Part of the Orthopedics Commons

Let us know how access to this document benefits you

\section{Recommended Citation}

Tischler, Eric H; Cavanaugh, Priscilla K; and Parvizi, Javad MD, "Leukocyte esterase strip test: matched for musculoskeletal infection society criteria." (2014). Rothman Institute Faculty

Papers. Paper 58.

https://jdc.jefferson.edu/rothman_institute/58

This Article is brought to you for free and open access by the Jefferson Digital Commons. The Jefferson Digital Commons is a service of Thomas Jefferson University's Center for Teaching and Learning (CTL). The Commons is a showcase for Jefferson books and journals, peer-reviewed scholarly publications, unique historical collections from the University archives, and teaching tools. The Jefferson Digital Commons allows researchers and interested readers anywhere in the world to learn about and keep up to date with Jefferson scholarship. This article has been accepted for inclusion in Rothman Institute Faculty Papers by an authorized administrator of the Jefferson Digital Commons. For more information, please contact: JeffersonDigitalCommons@jefferson.edu. 


\title{
Leukocyte Esterase Strip Test: Matched for Musculoskeletal Infection Society Criteria
}

\author{
Eric H. Tischler, BA, Priscilla K. Cavanaugh, MS, and Javad Parvizi, MD, FRCS \\ Investigation performed at The Rothman Institute at Thomas Jefferson Hospital, Thomas Jefferson University, Philadelphia, Pennsylvania
}

\begin{abstract}
Background: The presence of leukocyte esterase in the synovial fluid has recently been proposed as a marker for periprosthetic joint infection. However, the sensitivity and specificity of leukocyte esterase has not been determined when matched for the current, most inclusive Musculoskeletal Infection Society (MSIS) criteria for periprosthetic joint infection.

Methods: The presence of leukocyte esterase was prospectively evaluated in synovial joint aspirates from hips and knees from May 2009 to May 2013. The cohort consisted of 189 hip and knee aspirations (fifty-two positive and 137 negative for infection). If the aspirate was bloody, a centrifuge was used to precipitate red blood cells and obtain clear synovial fluid. A standard chemical test strip (graded as negative, trace, + , or ++ ) was used to detect the presence of leukocyte esterase. The sensitivity, specificity, positive predictive value, and negative predictive value of the leukocyte esterase strip test were calculated using ++ and $++/+$ as two positive strip result scenarios.
\end{abstract}

Results: Synovial fluid was obtained from 221 joints that underwent revision total hip or total knee arthroplasty for either mechanical failure or periprosthetic infection. Due to the lack of adequate criteria for MSIS criteria classification, thirty-two joints were excluded. The leukocyte esterase test with a threshold of $+/++$ had a sensitivity, specificity, positive predictive value, and negative predictive value of $79.2 \%$ (95\% confidence interval [Cl], $65.9 \%$ to $89.2 \%), 80.8 \%(95 \% \mathrm{Cl}$, $73.3 \%$ to $87.1 \%), 61.8 \%(95 \% \mathrm{Cl}, 49.2 \%$ to $73.3 \%)$, and $90.1 \%(95 \% \mathrm{Cl}, 84.3 \%$ to $95.4 \%)$, respectively. Using the ++ as a positive leukocyte esterase result, the sensitivity, specificity, positive predictive value, and negative predictive value were $66.0 \%$ ( $95 \% \mathrm{Cl}, 51.7 \%$ to $78.5 \%$ ), $97.1 \%$ ( $95 \% \mathrm{Cl}, 92.6 \%$ to $99.2 \%), 89.7 \%$ (95\% Cl, $75.8 \%$ to $97.1 \%$ ), and $88.0 \%(95 \% \mathrm{Cl}$, $81.7 \%$ to $92.7 \%)$, respectively.

Conclusions: When matched to the current MSIS criteria, the leukocyte esterase strip test yielded a high specificity, positive predictive value, negative predictive value, and moderate sensitivity. These results demonstrate that leukocyte esterase is an accurate, effective marker of periprosthetic joint infection as defined by the MSIS criteria. The leukocyte esterase strip test is a valuable tool that can be used in conjunction with the current battery of diagnostic tests available.

Level of Evidence: Diagnostic Level II. See Instructions for Authors for a complete description of levels of evidence.

Peer Review: This article was reviewed by the Editor-in-Chief and one Deputy Editor, and it underwent blinded review by two or more outside experts. It was also reviewed by an expert in methodology and statistics. The Deputy Editor reviewed each revision of the article, and it underwent a final review by the Editor-in-Chief prior to publication. Final corrections and clarifications occurred during one or more exchanges between the author(s) and copyeditors.

$\mathrm{P}$ eriprosthetic joint infection is one of the most serious complications after total joint arthroplasty. An estimated $1 \%$ to $3 \%$ of patients who undergo total joint arthroplasty subsequently develop a periprosthetic joint infection ${ }^{1-3}$. Revision arthroplasty is associated with an even higher prevalence of periprosthetic joint infection for knees and hips, $5.6 \%$ and $3.2 \%$, respectively ${ }^{4}$. In revision arthroplasty, it is vital to preoperatively distinguish between septic and aseptic failure because the two require different treatment.

The lack of a standard for the diagnosis of periprosthetic joint infection continues to pose challenges in the orthopaedic community ${ }^{5}$. In an effort to provide a universal standard

Disclosure: None of the authors received payments or services, either directly or indirectly (i.e., via his or her institution), from a third party in support of any aspect of this work. One or more of the authors, or his or her institution, has had a financial relationship, in the thirty-six months prior to submission of this work, with an entity in the biomedical arena that could be perceived to influence or have the potential to influence what is written in this work. No author has had any other relationships, or has engaged in any other activities, that could be perceived to influence or have the potential to influence what is written in this work. The complete Disclosures of Potential Conflicts of Interest submitted by authors are always provided with the online version of the article. 
TABLE I Comparison of the Mean Results on Laboratory Tests for the Cohorts with Positive and Negative Findings for Infection According to MSIS Criteria*

\begin{tabular}{|lcrr|}
\hline & \multicolumn{4}{c|}{ Mean Values } \\
\cline { 2 - 4 } Laboratory Test $\dagger$ & MSIS-Negative Cohort $(\mathrm{N}=137)$ & MSIS-Positive Cohort $(\mathrm{N}=52)$ & P Value \\
\hline ESR $(\mathrm{mm} / \mathrm{hr})$ & 24.4 & 40.40 & $<0.001$ \\
CRP level $(\mathrm{mg} / \mathrm{dL})$ & 0.96 & 3.22 & 0.001 \\
WBC count $(\mathrm{cell} / \mathrm{s} / \mu \mathrm{L})$ & $11,511.30$ & $32,795.33$ & $<0.001$ \\
PMN percentage & 51.51 & 84.54 & $<0.001$ \\
\hline
\end{tabular}

*MSIS = Musculoskeletal Infection Society. $†$ ESR $=$ erythrocyte sedimentation rate, $\mathrm{CRP}=\mathrm{C}$-reactive protein, $\mathrm{WBC}=$ white blood cell, and $\mathrm{PMN}=$ polymorphonuclear.

definition of periprosthetic joint infection, the work group of the Musculoskeletal Infection Society (MSIS) developed diagnostic criteria for periprosthetic joint infection ${ }^{6}$, which were recently endorsed and updated by an International Consensus Group on Periprosthetic Joint Infection ${ }^{7}$. However, the search for a single diagnostic test that possesses sufficient reliability, accuracy, sensitivity, and specificity continues. The need for a more accurate and efficient test for the diagnosis of periprosthetic joint infection led to the introduction of leukocyte esterase strip testing of synovial fluid aspirates at our institution ${ }^{8}$.

Leukocyte esterase is an enzyme secreted by activated neutrophils that have been recruited to areas of infection ${ }^{8}$. In periprosthetic joint infection, neutrophils recruited to the joint secrete leukocyte esterase that is detectable by colorimetric strip tests through reactions that result in a color change and that vary according to the type of strip used ${ }^{8}$. This simple test requires application of synovial fluid to a urine test strip with subsequent attention to leukocyte esterase ${ }^{6}$. Parvizi et al. ${ }^{8}$ demonstrated that the leukocyte esterase strip test may have a valuable role in the diagnosis of periprosthetic joint infection, yielding a sensitivity of $80.6 \%$ and a specificity of $100 \%$ for $\mathrm{a}++$ strip reading. The leukocyte esterase strip test is a quick, easy-to-perform, cost-effective measure with high sensitivity and specificity. Similarly, Wetters et al. ${ }^{9}$ found the leukocyte esterase strip test valuable with a sensitivity and specificity ranging from $92.9 \%$ to $100 \%$ and $77.0 \%$ to $88.8 \%$, respectively, for the detection of periprosthestic joint infection. The ranges were due to the use of different indicators of periprosthetic joint infection (synovial white blood cell count, positive cultures, and reoperation), which the authors noted as a limitation since different definitions of periprosthetic joint infection could lead to different results?.

This prospective study was designed to test the utility of the leukocyte esterase strip test for diagnosis of periprosthetic joint infection when infection was defined using the inclusive MSIS criteria. Prior literature on the leukocyte esterase test revealed high sensitivity and specificity; however, those results were based on a variety of periprosthetic joint infection definitions. To our knowledge, this is the first study evaluating the role of the leukocyte esterase strip test for diagnosing periprosthetic joint infection as defined by the current MSIS cri- teria. Additionally, in the February 2014 Proceedings of the International Consensus on Periprosthetic Joint Infection, it was reported that the group voted to include the ++ leukocyte esterase reading as part of the minor MSIS criteria, further indicating the importance and relevance of this test metric ${ }^{7}$.

\section{Materials and Methods}

n approval of the institutional review board, which allows for surgical waste product to be collected without patient consent, we prospectively performed the leukocyte esterase strip test on 221 consecutive patients who had revision total knee or hip arthroplasty for either aseptic failure or periprosthetic infection from March 2009 to May 2013. Of the 221 revision arthroplasties, thirty-two patients were excluded because of the lack of adequate criteria for MSIS classification.

There were 189 patients in the final cohort; ninety-nine $(52.4 \%)$ of them were women and ninety $(47.6 \%)$ were men. Thirty-five hip and 154 knee arthroplasty revisions were included. The cohort had a mean age and body mass index (BMI) of sixty-three years (range, twenty-one to ninety years) and $32.9 \mathrm{~kg} / \mathrm{m}^{2}$ (range, 21.1 to $54.2 \mathrm{~kg} / \mathrm{m}^{2}$ ), respectively. Mean serum erythrocyte sedimentation rate (ESR), serum C-reactive protein (CRP) level, synovial fluid white blood-cell (WBC) count, and synovial fluid polymorphonuclear (PMN) percentage were reported for both the MSIS positive and negative cohorts (Table I).

We obtained intraoperative synovial fluid from joints undergoing revision total knee and hip arthroplasty. Synovial aspiration was performed just prior to the arthrotomy, with use of an 18-gauge needle. This was done to minimize blood contamination of the synovial fluid that interferes with the leukocyte esterase test. If the synovial fluid had any contaminant, a minimum of $1.5 \mathrm{~mL}$ of synovial fluid was transferred to one or more Sarstedt Micro centrifuge tubes (Sarstedt, Nümbrecht, Germany). These tubes were then placed in a Fisherbrand Mini Centrifuge (dimensions, $16.2 \times 15.9 \times 10.2 \mathrm{~cm}$; Fisher Scientific, Waltham, Massachusetts). The contaminated samples were spun at maximum speed for 120 to 180 seconds, after which synovial fluid could be extracted and isolated from nonsynovial fluid, typically, erythrocytes. Aggarwal et al. previously noted that the use of the centrifuge does not compromise the accuracy or affect the result of the leukocyte esterase reading ${ }^{10}$. Needle aspiration of this isolated supernatant synovial fluid allows for accurate colorimetric strip testing using the Chemstrip 7 urine test strip (Roche Diagnostics, Indianapolis, Indiana).

Immediately following aspiration, one drop of synovial fluid was applied to the leukocyte test pad of two standard chemical test strips to detect the presence of leukocyte esterase. Results were recorded after sixty to 120 seconds. Two leukocyte esterase strips were used per aspiration to ensure the reliability of the strip result. For each individual synovial fluid sample, the two leukocyte esterase strips used always had the same reading (negative, trace, + , or ++ ). Leukocyte esterase strip results were analyzed directly in the operating room or 
The Journal of Bone \& Joint Surgery • Jbjs. org Volume 96-A • Number 22 - November 19, 2014

\begin{tabular}{|lc|}
\hline TABLE II Organisms Found in Patients with Positive Cultures \\
\hline Culture-Positive Organism & $\begin{array}{c}\text { No. of Patients } \\
\text { Identified } \\
\text { with Organism }\end{array}$ \\
\hline Coagulase-negative Staphylococcus & 7 \\
Staphylococcus aureus & 4 \\
Enterococcus faecalis group D & 1 \\
Corynebacterium jeikeium & 1 \\
Beta-hemolytic Streptococci group G & 1 \\
Coagulase-negative Staphylococcus & 1 \\
and/or Staphylococcus lugdunensis & 1 \\
Actinomyces israelii & \\
\hline
\end{tabular}

\begin{tabular}{|c|c|c|}
\hline Measurement Outcome & Result & 95\% Confidence Interval \\
\hline \multicolumn{3}{|l|}{$+/++$ Threshold } \\
\hline Sensitivity (\%) & 79.2 & $65.9-89.2$ \\
\hline Specificity (\%) & 80.8 & 73.3-87.1 \\
\hline PPV (\%) & 61.8 & $49.2-73.3$ \\
\hline NPV (\%) & 90.1 & 84.3-95.4 \\
\hline \multicolumn{3}{|l|}{++ Threshold } \\
\hline Sensitivity (\%) & 66.0 & $51.7-78.5$ \\
\hline Specificity (\%) & 97.1 & $92.6-99.2$ \\
\hline PPV (\%) & 89.7 & $75.8-97.1$ \\
\hline NPV (\%) & 88.0 & $81.7-92.7$ \\
\hline
\end{tabular}

*MSIS = Musculoskeletal Infection Society, PPV = positive predictive value, NPV = negative predictive value.

in the office setting. In either circumstance, results were read and interpreted by trained orthopaedic research fellows. The color of the test strip was read as negative (white), trace (slightly purple), + (light purple), or ++ (dark purple).

Each joint was then classified as positive or negative for periprosthetic joint infection using the MSIS infection criteria ${ }^{6}$ :

- There is a sinus tract communicating with the prosthesis; or

- A pathogen is isolated by culture from at least two separate tissue or fluid samples obtained from the affected prosthetic joint; or

- Three of the following five criteria exist:

- Elevated serum ESR ( $\geq 30 \mathrm{~mm} / \mathrm{hr}$ ) and serum CRP concentration $(\geq 10 \mathrm{mg} / \mathrm{L})$

- Elevated synovial WBC count $(\geq 2000 / \mu \mathrm{L})$

- Elevated synovial fluid PMN percentage ( $\geq 65 \%)$

- Presence of purulence in the affected joint

- Isolation of microorganism in one culture of periprosthetic tissue or fluid

\section{Statistical Analysis}

We calculated the sensitivity, specificity, positive predictive value, and negative predictive value of the leukocyte esterase strip test in diagnosing periprosthetic
Leukocyte Esterase Strip Test: Matched for

Musculoskeletal Infection Society Criteria joint infection using the MSIS criteria as the standard. The statistical analysis was conducted in two parts: (1) a ++ reading represented a positive result, whereas a negative, trace, and + reading were all considered negative for infection and (2) a combined $+1++$ represented a positive result-i.e., either a + result or a ++ result was considered positive for leukocyte esterase, rather than just a ++ result-while negative and trace were considered negative.

\section{Source of Funding}

There was no external funding.

\section{Results}

$\mathrm{f}$ the fifty-two patients who were considered to have an infection according to the MSIS criteria, sixteen had positive cultures and thirty-six had negative cultures. Table II provides a distribution of the organisms identified for patients with a positive culture. At our institution, all coagulase-negative Staphylococcus organisms are categorized together.

Of the 221 revision hip and knee joints aspirated, thirtytwo (14.5\%) did not have adequate testing to allow evaluation of the MSIS criteria and were ultimately excluded. Of the thirty-two patients excluded, $12.5 \%$ (four) had incomplete culture data, 6.3\% (two) had incomplete serum data, and $81.3 \%$ (twenty-six) had incomplete synovial fluid data, either as a result of inadequate fluid sample to perform all tests or a dry tap.

Of the remaining 189 patients, the leukocyte esterase test was read as negative for eighty-three (44\%), trace for thirtyeight $(20 \%),+$ for twenty-nine (15\%), and ++ for thirty-nine patients $(21 \%)$.

When using the MSIS infection criteria as the so-called gold standard for the diagnosis of periprosthetic joint infection, the leukocyte esterase test with a threshold of $+/++$ had a sensitivity, specificity, positive predictive value, and negative predictive value of $79.2 \%$ (95\% confidence interval $[\mathrm{CI}], 65.9 \%$ to $89.2 \%$ ), $80.8 \%$ (95\% CI, $73.3 \%$ to $87.1 \%$ ), $61.8 \%$ (95\% CI, $49.2 \%$ to $73.3 \%$ ), and $90.1 \%$ (95\% CI, $84.3 \%$ to $95.4 \%$ ), respectively (Table III). Using the ++ as a positive leukocyte esterase result, the sensitivity, specificity, positive predictive value, and negative predictive value were $66.0 \%$ (95\% CI, $51.7 \%$ to $78.5 \%$ ), $97.1 \%$ (95\% CI, $92.6 \%$ to $99.2 \%$ ), $89.7 \%$ ( $95 \% \mathrm{CI}, 75.8 \%$ to $97.1 \%$ ), and $88.0 \%$ (95\% CI, $81.7 \%$ to 92.7\%) (Table III).

\section{Discussion}

The lack of a universal definition of periprosthetic joint infection has made its diagnosis a constant challenge. In an effort to create a more accurate and universal set of diagnostic criteria, the MSIS proposed an inclusive definition of periprosthetic joint infection ${ }^{6}$ with the intention to eliminate discrepancies between various infection criteria. The adoption of a common set of diagnostic criteria, although a major step, is only part of the solution to achieving optimal diagnostic accuracy and efficiency. The need for a rapid, accurate, costeffective marker for periprosthetic joint infection remains.

The shortcomings of a single diagnostic test for periprosthetic joint infection directed our work toward leukocyte esterase $^{8}$. In a prior study, Parvizi et al. ${ }^{8}$ demonstrated that the 
The Journal of Bone \& Joint Surgery JbjS. org Volume 96-A • Number 22 - November 19, 2014
Leukocyte Esterase Strip Test: Matched for

Musculoskeletal Infection Society Criteria leukocyte esterase strip had a relatively high sensitivity and specificity for detecting periprosthetic joint infection, later supported by Wetters et al. ${ }^{9}$. Furthermore, leukocyte esterase results correlated substantially with four parameters typically used to screen for periprosthetic joint infection (ESR, CRP, WBC count, and synovial fluid PMN percentage $)^{8}$. Together, these five parameters could suggest a useful clinical diagnosis of periprosthetic joint infection.

The purpose of our study was to evaluate the usefulness of the leukocyte esterase strip test in accurately diagnosing periprosthetic joint infection as defined by the MSIS criteria. Our results indicate that the leukocyte esterase strip test remains a valuable diagnostic test for periprosthetic joint infection when using the MSIS criteria to define periprosthetic joint infection. Leukocyte esterase demonstrated a high specificity, negative predictive value, and positive predictive value, although the sensitivity was not as high as previously reported (Table III). Although our data do not support the use of leukocyte esterase as an independent screening method because of a less than optimal sensitivity, the extremely high specificity and negative predictive value in conjunction with other parameters make it an extremely efficient test to rule out infection. Additionally, it should be noted that positive predictive value results are prevalence dependent, meaning that if the prior probability of infection were very low, then the positive predictive value would be much lower, even with the same reported sensitivity and specificity. Given these findings, this would indicate that a trace or negative leukocyte esterase should allow the surgeon to rule out a periprosthetic infection with relative certainty. The leukocyte esterase strip test is an important addition to the group of diagnostic tests available. Its rapid results (one minute), ease of use, and low cost allow it to be used both in outpatient and intraoperative settings.

Similar to other current diagnostic methods, the leukocyte esterase strip test has limitations. Any debris or blood in the synovial aspirate has the potential to skew the results of the colorimetric test, as exemplified in one-third of the aspirates that could not be tested with colorimetric reagents strips in the study by Wetters et al. ${ }^{9}$. We addressed this concern by using centrifugation to separate the bloody contaminant from the synovial fluid in cases of bloody aspirate, a protocol that preserves the accuracy of the colorimetric test for leukocyte esterase $\mathrm{e}^{10}$. Although all individuals were trained to evaluate the leukocyte esterase strip test results, a possible bias is introduced because of the subjectivity of leukocyte esterase strip interpretation.

The future for leukocyte esterase as a marker for periprosthetic joint infection is promising. In revision arthroplasty, leukocyte esterase may be a more accurate and efficient predictor of infection at reimplantation than current markers such as ESR and CRP, which have a low specificity for periprosthetic joint infection ${ }^{11,12}$.

Leukocyte esterase may yield false-positive $(++)$ results in patients with aseptic loosening of metal-on-metal total hip replacements because of white blood-cell recruitment to the joint site of inflammation; however, none of the patients with aseptic loosening of metal-on-metal implants in our cohort yielded ++ results, suggesting that leukocyte esterase is still valid in this circumstance. Nevertheless, further research is required to evaluate the true effects of metal-on-metal total hip replacement tissue reactions and leukocyte esterase.

In conclusion, detection of leukocyte esterase in synovial fluid appears to be a highly accurate, simple, rapid, and valuable tool for the diagnosis of periprosthetic joint infection, demonstrating a high specificity and negative predictive value for the diagnosis of periprosthetic joint infection when applied to the MSIS criteria.

Eric H. Tischler, BA

Priscilla K. Cavanaugh, MS

Javad Parvizi, MD, FRCS

The Rothman Institute at Thomas Jefferson Hospital,

Thomas Jefferson University,

Sheridan Building,

10th Floor, 125 South 9th Street,

Philadelphia, PA 19107.

E-mail address for J. Parvizi: parvj@aol.com

\section{References}

1. Phillips JE, Crane TP, Noy M, Elliott TS, Grimer RJ. The incidence of deep prosthetic infections in a specialist orthopaedic hospital: a 15-year prospective survey. J Bone Joint Surg Br. 2006 Jul;88(7):943-8.

2. Kurtz SM, Lau E, Schmier J, Ong KL, Zhao K, Parvizi J. Infection burden for hip and knee arthroplasty in the United States. J Arthroplasty. 2008 Oct;23(7):984-91. Epub 2008 Apr 10.

3. Jämsen $\mathrm{E}$, Varonen $\mathrm{M}$, Huhtala $\mathrm{H}$, Lehto MU, Lumio J, Konttinen YT, Moilanen T. Incidence of prosthetic joint infections after primary knee arthroplasty. J Arthroplasty. 2010 Jan;25(1):87-92. Epub 2008 Dec 04.

4. Hanssen AD, Rand JA. Evaluation and treatment of infection at the site of a total hip or knee arthroplasty. Instr Course Lect. 1999;48:111-22.

5. Bauer TW, Parvizi J, Kobayashi N, Krebs V. Diagnosis of periprosthetic infection. J Bone Joint Surg Am. 2006 Apr;88(4):869-82.

6. Parvizi J, Zmistowski B, Berbari EF, Bauer TW, Springer BD, Della Valle CJ, Garvin KL, Mont MA, Wongworawat MD, Zalavras CG. New definition for periprosthetic joint infection: from the Workgroup of the Musculoskeletal Infection Society. Clin Orthop Relat Res. 2011 Nov;469(11):2992-4.
7. Parvizi J, Gehrke T; International Consensus Group on Periprosthetic Joint Infection. Definition of periprosthetic joint infection. J Arthroplasty. 2014 Jul;29 (7):1331. Epub 2014 Mar 21.

8. Parvizi J, Jacovides C, Antoci V, Ghanem E. Diagnosis of periprosthetic joint infection: the utility of a simple yet unappreciated enzyme. J Bone Joint Surg Am. 2011 Dec 21;93(24):2242-8.

9. Wetters NG, Berend KR, Lombardi AV, Morris MJ, Tucker TL, Della Valle CJ. Leukocyte esterase reagent strips for the rapid diagnosis of periprosthetic joint infection. J Arthroplasty. 2012 Sep;27(8)(Suppl):8-11. Epub 2012 May 17.

10. Aggarwal VK, Tischler E, Ghanem E, Parvizi J. Leukocyte esterase from synovial fluid aspirate: a technical note. J Arthroplasty. 2013 Jan;28(1):193-5. Epub 2012 Aug 03.

11. Bedair H, Ting N, Jacovides C, Saxena A, Moric M, Parvizi J, Della Valle CJ. The Mark Coventry Award: diagnosis of early postoperative TKA infection using synovial fluid analysis. Clin Ortop Relat Res. 2011 Jan;469(1):34-40.

12. Parvizi J, Ghanem E, Menashe S, Barrack RL, Bauer TW. Periprosthetic infection: what are the diagnostic challenges? J Bone Joint Surg Am. 2006 Dec; 88(Suppl 4):138-47. 